\title{
Micro Grid Feasibility - A Multi-Scaling Approach
}

\author{
Dr Valsamma KM \\ Associate Professor, Department Of SAC, Faculty of Agricultural Engineering, Kerala Agricultural University, \\ KCAET, Tavanur-679573,
}

\begin{abstract}
Rural and Remote electrification is now-a day's an important market for renewable energy production and since the last decade, off-grid rural electrification with renewable energy sources has become a cost-effective option. This study presents an analysis for choosing the best hybrid Renewable Energy Technology system \& estimates systematically the dynamics of electrical power generation from a mix of renewable energy, to satisfy the needs of the rural area Kuttumpuzha in India, using multi-scaling analysis. In order to demonstrate the capability of fractal approach on electrical power demand, we choose Wavelet Transform Modulus maxima (WTMM) technology and results imply that this method is powerful in studying the singularities of time series signals. We show that the WTMM, the so called multi-resolution analysis, can determine the singularity spectrum of multi-fractal measures from the scaling behavior of the partition function, and account for the multi-fractal nature of the time series obtained from the chaotic phenomena. Applying the WTMM we will be able to verify that the time series of micro grid incorporated with renewable energy resources is a persistent series and this method can be used to compare the quality of the process using different mix of renewable resources. The correlation dimensions found tells us that the series obtained is long-range correlated with long term memory. This lays that a hybrid renewable energy technology system with a combination of solar, \& wind, energy system, converter, and batteries, for the rural village from the different perspective combinations for power generating system is cost effective even in areas where the grid connection would be possible and better suited to satisfy oscillations in the demands .
\end{abstract}

Keywords - Renewable Energy Resources, Photo voltaic cells, Wavelet Transform Modulus Maxima, Multi-Scaling, Lipchitz holder exponent, Singularity spectrum, Fractal Dimension..

\section{INTRODUCTION}

Due to industrialization, the demand for energy for various purposes has been increasing rapidly not only in India, but over the whole world. Energy security and sustainable development are high on the global agenda due to the impact of volatile energy prices, high demand of energy, and concerns over environmental sustainability and the global climate change. Conventional energy resources alone will not be able to meet the ever increasing energy demand, New and renewable energy technologies (RET) are considered to be one of the viable options to meet the challenge of achieving sustainable development, while conserving natural resources that have been depleted due to the rapid growth in population, urbanization and fossil fuel consumption.

The renewable electricity generation capacity reached an estimated 240GW worldwide in 2007 which represents the $5 \%$ of the global power capacity and $3.4 \%$ of the global power generation. The Indian renewable energy (RE) sector has shown impressive growth in the past few years and investments in the sector have increased significantly. The share of renewable was $7.7 \%$ in the cumulative installed capacity $1147403 \mathrm{MW}$ of electric power plants under utilities in India until 2008 and by 2012 expects to contribute 10\% of the total power generation capacity with 4\%-5\% share in the energy mix. The cumulative installed capacity of grid-interactive RE systems for power generation was 13879 MW until 2009 of which contribution of wind, small hydro, bio-mass and solar were $9756,2345,1776, \& 2.12 \mathrm{MW}$ respectively[1], with wind power contributing $70 \%$ of the grid-interacting renewable power installed in the country. With an electricity demand of $322 \mathrm{KWh}$ per capita per year in 2003, India's electricity demand has been growing at an average of $8.8 \%$ per year from 1986 tom 2003 while the peak demand increased on average by $6.3 \%$ per annum from $540 \mathrm{MW}$ to $1516 \mathrm{MW}$. If we look at the consumption electricity, per capita consumption in India is far below that in other countries with. access to electricity being uneven. In 2003, about $68 \%$ of Indian households were connected to the electricity grid with household electricity consumption accounting for about $35 \%$ of total electricity consumption, and household and Industrial sector consumption combined accounting for $65 \%$ of the total $6.209 \mathrm{GWH}$. India as a developing country, where majority of the population lives in rural areas with no access to electricity, extension of the central electricity grid is not a viable or 
Micro Grid Feasibility - A Multi-Scaling Approach

practically feasible solution, but hybrid power systems involving one or more RE based generating units such as solar and wind generating units, combined with battery backups can satisfy the load demand.

A new method to quantify intricate patterns of time series signals, as projections of fractal functions with deterministic nature, and which go through multi- scaling is the Multi-fractal analysis. Various multi-fractal formalism have recently been developed to describe the statistical properties of the singular measure of signals in terms of their singularity spectrum $f(\propto)$, or generalized dimension $D_{q}$.. The idea of distribution of singularities all lying on interwoven sets of varying fractal dimensions called multi-fractal was introduced by Frish etal [2].Further, Hentschel etal [3] introduced the Generalized dimension $D_{q}$, and Halsey etal [4] the $f(\propto c)$ spectrum. Wavelets are a recently developed concept and WTMM method [5] with continuous basis function, is a well known method to investigate the multi-fractal scaling properties of fractal and self affine objects in the presence of non stationeries. In this paper, we intend to clarify the characteristics of electrical power demand using WTMM with continuous function.

\section{METHODOLOGY}

\section{Multi-fractal Analysis of Non-Linear Time Series using wavelet method:}

A time series, is a sequence of scalar measurements of some quantity, which depends on the current state of the system, taken at multiples of fixed sampling time $(\Delta t)$. To reveal the multi-dimensional aspects of the system, phase space reconstruction of the time series is required, which requires proper choice of the embedding dimension (m) and delay time $(\tau)$. If the time delayed mutual information shows a marked minimum, this signifies a reasonable time delay $(\tau)$.Using embedding theorem, one can reconstruct a multi dimensional state space $Y_{n}=\left(X_{(n-m+1)} \tau, X_{(n-}\right.$ $\left.{ }_{\mathrm{m}+2)} \tau, \ldots \ldots ., \mathrm{X}_{(\mathrm{n}-} \tau, \mathrm{X}_{\mathrm{n}}\right)$ with vectors $\mathrm{Y}_{\mathrm{n}}($ for $\mathrm{n}=1,2,3 \ldots$.$) . and can quantify deterministic chaos, statistically, using$ quantifiers like Fractal Dimension $\mathrm{D}_{0}$, Correlation dimension $\mathrm{D}_{2}$, and Information dimension $\mathrm{D}_{1}$.

Multi-fractal signals are those endowed with the property of abrupt change in regularity from one point to another and often possess a self similar structure at varying levels of resolution, revealing finer details at arbitrary smaller scales. Finding the distribution of singularities in a multi-fractal signal is particularly important for detecting, identifying, and .measuring the scaling behavior. The time -frequency localization property of Wavelet Transforms, allows it to be chosen as one of the best techniques for location of the various singularities in a signal. In order to measure the singularity spectrum of multi-fractal signals, we use the wavelet transform local maxima, using global partition function (for computing the attributes like Lipchitz holder exponent and singularity spectrum). .Mallat [5] has proved that all singularities of irregular signal (multi-fractal signal) could be detected using WTMM in partition function and shown the method to measure the local singularity strength $\alpha$. Numerical analysis performed in our work was based on WTMM method [6 ].This is one of the commonly used approaches to study multi scale structures in complex time series. Using WTMM in calculating the partition function, we can avoid the deviations that are made by the oscillation of wavelet coefficients when $\mathrm{q}<0$. It is more accurate and efficient in detecting singularities in signal. That is, the attractiveness of using the WTMM is associated with the possibility it provides of analyzing a wide range of scales and a broad spectrum of scaling characteristics from small fluctuations and weak singularities to large fluctuations and strong singularities. In this approach, the numerical quantification of time series is done by the so called singularity spectrum $\mathrm{D}(\mathrm{h})$ characterized by the Holder exponent $\mathrm{h}$ (local measure characterizing or quantifying the strength/ smoothness of a distribution/measure).

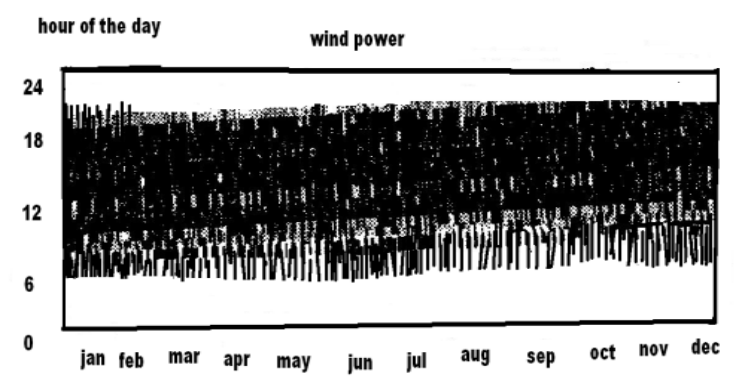

(a) 


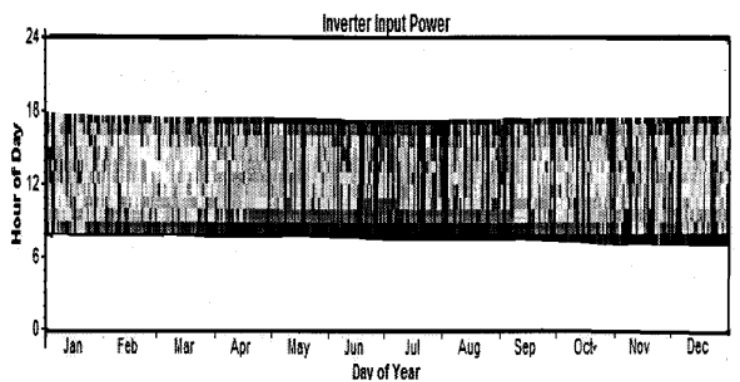

(b)

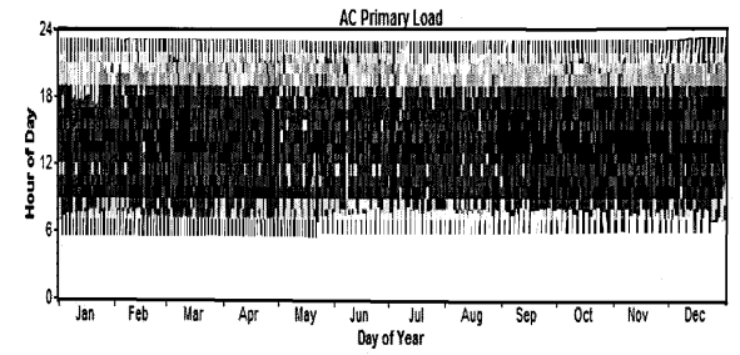

(c)

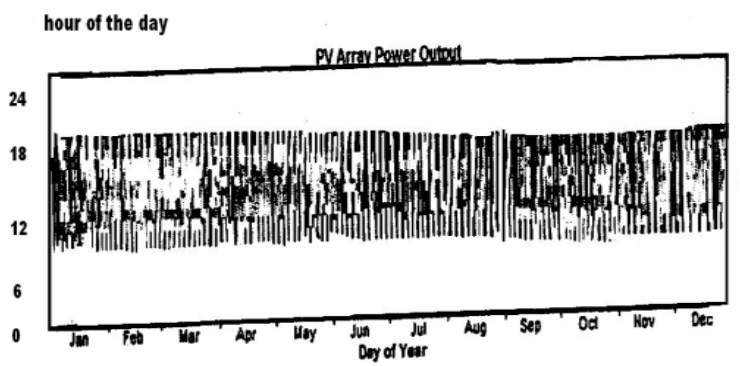

(d)

Fig 1.Density Profile for (a) Wind Turbine, (b)

Inverter. (c) AC primary load (d) Solar Photo voltaic.

For a continuous process with spectral density $\Gamma_{\mathrm{x}}(\vartheta)$, we define the wavelet transform of a function $\mathrm{f}(\mathrm{x})$ as:

$$
\mathrm{T} \Psi\left|\mathrm{f}\left(\mathrm{x}_{0}, \mathrm{a}\right)\right|=\frac{1}{a} \int_{-\infty}^{+\infty} f(x) \Psi\left(\frac{x-x 0}{a}\right) \mathrm{dx}
$$

Where $\mathrm{f}\left(\mathrm{x}_{0}\right)$ is a distribution at a point $\mathrm{x}_{0}$ (location parameter), a is the scale parameter, and $\Psi$ defines the family of wavelets. : that is, for varying values of a, wavelets of different length scales can be constructed. The wavelet transform can be seen as decomposing the function $\mathrm{f}(\mathrm{x})$ in to elementary space scale contributions by combining it with a suite of localized functions, the so called wavelets, all of which are constructed by translation and dilation of a single function, the analyzing wavelet. An attractive feature of wavelets is that one could construct various analyzing patterns which satisfy the requirements for a function to be called a wavelet. A local singular behavior of $\mathrm{f}(\mathrm{x})$ at the point $\mathrm{x}_{0}$ results in an increase of $|\mathrm{T} \Psi| \mathrm{f}(\mathrm{x}, \mathrm{a}) \|$ as $\mathrm{x} \rightarrow \mathrm{x}_{0}$, and can be characterized by the Hölder exponent $\mathrm{h}\left(\mathrm{x}_{0}\right)$ that quantifies the scaling of the wavelet coefficients for small a: $\mathrm{T} \Psi\left|\mathrm{f}\left(\mathrm{x}_{0}, \mathrm{a}\right)\right|^{\sim} \alpha^{\mathrm{T}(\mathrm{q})}$. Further, the statistical description of local singularities is performed using the notion of the partition function $\mathrm{S}(\mathrm{q}, \mathrm{a})$ [7], being the sum of the $\mathrm{q}^{\text {th }}$ powers of the local maxima of $\mathrm{T} \Psi|\mathrm{f}(\mathrm{x}, \mathrm{a})|$ at a scale $\alpha$. For small $\alpha$, the partition function $\mathrm{S}(\mathrm{q}, \mathrm{a})$ scales as $[8]$ :

$$
\mathrm{S}(\mathrm{q}, \mathrm{a})^{\sim} \alpha^{\mathrm{T}(\mathrm{q})} .
$$


with the scaling exponent ${ }^{\mathrm{T}(\mathrm{q})}$. The singularity spectrum $\mathrm{D}(\mathrm{h})$ (Global measure that computes the set of fractal dimensions as well as the hierarchy of singularities of a signal, specifying how often specific values of the singularity strength occur) can be estimated using the Legendre transform :

$$
\mathrm{D}(\mathrm{h})=\mathrm{q}(\mathrm{h})-\mathrm{T}(\mathrm{q})
$$

For positive values of $\mathrm{q}$, whereas the partition function $\mathrm{S}(\mathrm{q}, \mathrm{a})$ characterize the scaling of large fluctuations in the data (strong singularities), for negative values of $\mathrm{q}$, it reflects the weak singularities.

\section{DATA AND METHOD OF ANALYSIS}

Renewable energy source available at a location can differ considerably from site to site,(solar being dependent on latitude and climate; wind resource influenced by atmospheric circulation pattern combined with geographic aspect and hydro resource with location, topography and rainfall), which in turn influence the power that can be generated (and hence the hybrid systems economics and behavior.), and this is the vital aspect in developing the hybrid system. Kuttumpuzha, one of the remote rural village in Ernakulum district, Kerala, between $10.15^{\circ} \mathrm{N}$ Latitude and $76.7333333^{\circ} \mathrm{E}$ longitude. is ideal for hybrid systems since solar and wind energies are moderately available. It is a fact that, frequent wind currents in Kuttumpuzha makes the area suitable for hybrid micro- grid systems, in which a major fraction could be met from wind power. Further, wind turbines need strong and steady wind for efficient energy production, whereas photo-voltaic generators are versatile, in response to varying solar insulation. Data used for this study was acquired from the NASA satellite resource website ( solar resource from surface meteorology and solar energy satellite data). In the measurement side, the average electricity demand of a typical house, in the region, was computed both from KSEB records and Watt meter readings, taking into account the initial cost, maintenance cost, and replacement value of circuit components as inputs. The electricity meter readings collected for ten days taken at one hour intervals. is used to calculate the electricity usage / hour, The hourly daily electricity usage profile (FIG.2) is mapped into bi-monthly electricity data and the average electricity usage for 20 houses over each month is calculated. The ratio of electricity use of each hour to the total electricity use of the day was found from the hourly usage curve. Multiplied with each months average daily electricity use, provides hourly usage for all days of the year .

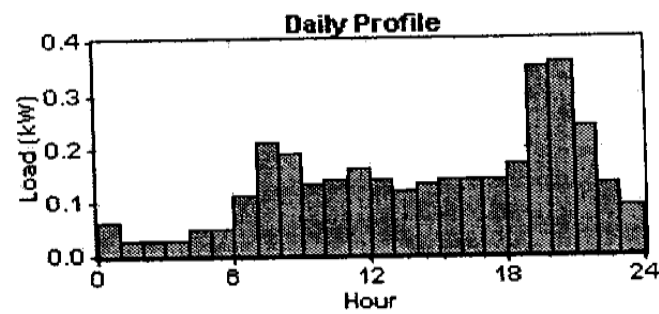

Fig.2,Hourly daily electricity usage profile.

Various combinations of different. RETS for off-grid electrifications of remote rural village, are taken for a detailed study. Solar Resource profile, (with clearness index 0.506 scaled down to $4.96 \mathrm{KWh} / \mathrm{m} 2 /$ day of annual solar radiation) and and wind resource (with annual average wind speed $3.59 \mathrm{~m} / \mathrm{sec}$ ), available for a span of one year is shown in figs(3-4).. The technical and cost parameters of each of the systems components are based on the data from MNRE AND TERI etc.The cost curve including mounting, hardware and installation and power curve for the PV of varying sizes, connected to DC output for a span of 20 years, wind Turbine with hub height $25 \mathrm{~m}$ (cost includes tower cost, controller, wiring, installation) with life span of 20 years, Converter and battery systems is given in Figs .5(a)-(d). 


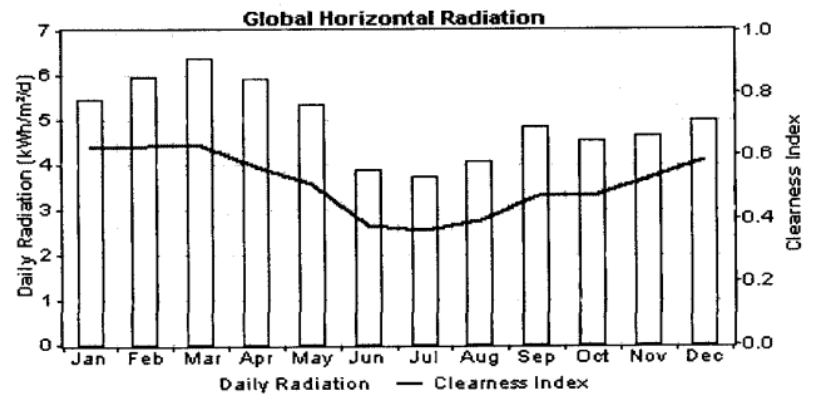

FIG.3.. Solar Resource profile for a span of one year.

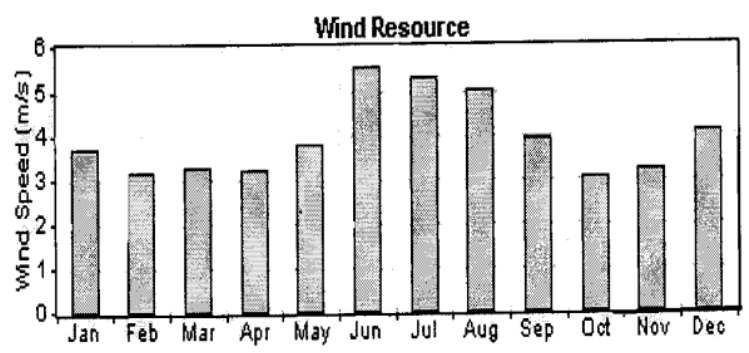

FIG.4..Wind Resource profile for a span of one year.

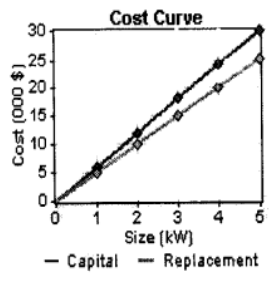

(a)

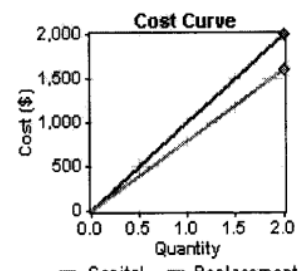

(c)

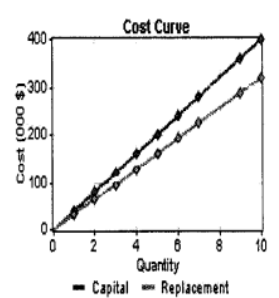

(b)

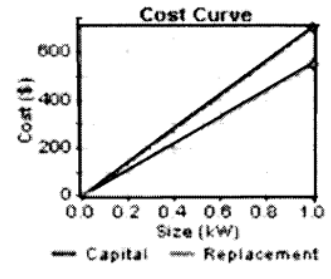

(d)

Fig.5. Technical and cost parameters for : (a) solar PV (b) Wind Turbine (c) Battery (d) inverter

Application of WTMM method to an analysis of point processes extracted from the time series of different combinations of hybrid RE systems, with 8760 points has revealed differences in the long range correlations, revealing that power generating system consisting of a combination of $1 \mathrm{KW}$ photo voltaic Array coupled with $1 \mathrm{KW}$ converter along with grid connection is cheap and is better suited to satisfy correlations in the demand. In the analysis, we use the successive derivatives of a Gaussian function( i.e., the the fifth derivative of Gaussian function was chosen) as analyzing wavelet:

$$
\Psi^{5}(\mathrm{t})=\mathrm{d}^{5}\left(\mathrm{e}^{\mathrm{t} 2 / 2}\right) / \mathrm{dt}^{5} ;
$$


Twelve wavelet transform data files were obtained applying the wavelet transform to solar PV data, wind energy data, electrical power grid, and storage battery data of Kuttumpuzha region, with $\Psi^{5}$, ranging the scaling factor $\alpha$ from $\alpha_{\min }=\frac{1}{256}$ to $\alpha_{\max }=8$ in steps of a power of two. The partition function for each one in the range $-20 \leq \mathrm{q} \leq$ 20 is computed. It is seen that $\tau(\mathrm{q})$ is a nonlinear convex increasing function with $\tau(0)=-0.65$ and the slopes $\alpha_{\text {min }}$ $=0.66$ for $\mathrm{q} \leq 0$ and $\alpha_{\max }=0.85$ for solar PV, while for wind turbine $\tau(0)=-0.54$ and $\alpha_{\min }=0.62$ for $\mathrm{q} \leq 0$ and $\alpha$ $\max =0.90$.The corresponding singularity spectra $\mathrm{D}(\mathrm{h})$, a single humped function with a non unique Holder exponent, are obtained by Legendre transforming $\tau(\mathrm{q})$ ( Fig.6.(a) - (d)). As expected from $\tau(\mathrm{q})$, the support of the $\mathrm{D}(\mathrm{h})$ extends over a finite interval whose bounds are $\alpha_{\min }=0.66$ and $\alpha_{\max }=0.84$ for solar PV which is larger than the one for Wind Turbine ranging from $\alpha_{\min }=0.62$ and $\alpha_{\max }=0.90$. The minimum value $\alpha_{\min }$, corresponds to the strongest singularity characterizing the most rarefied zones, while higher values exhibit weaker singularities until $\alpha$ max which corresponds to the densest zone. $\alpha_{\max \&} \alpha_{\min }$ both between $0.5 \& 1$ signifies persistent processes, although a very little less persistence for the case of Solar PV than Wind turbine (highly persistent) due to slight shift of the curve to the right. Further for, battery storage system, the observed d(h)-h curve shows a much broader spectrum with the left hump slightly raised above the right one, depicting availability of energy over high values of $h$. In the case of inverter output $\mathrm{D}(\mathrm{h})$-h curve is evenly distributed showing availability of energy output evenly distributed at all times. However, the analyzed data remain multi-fractal at all stages.
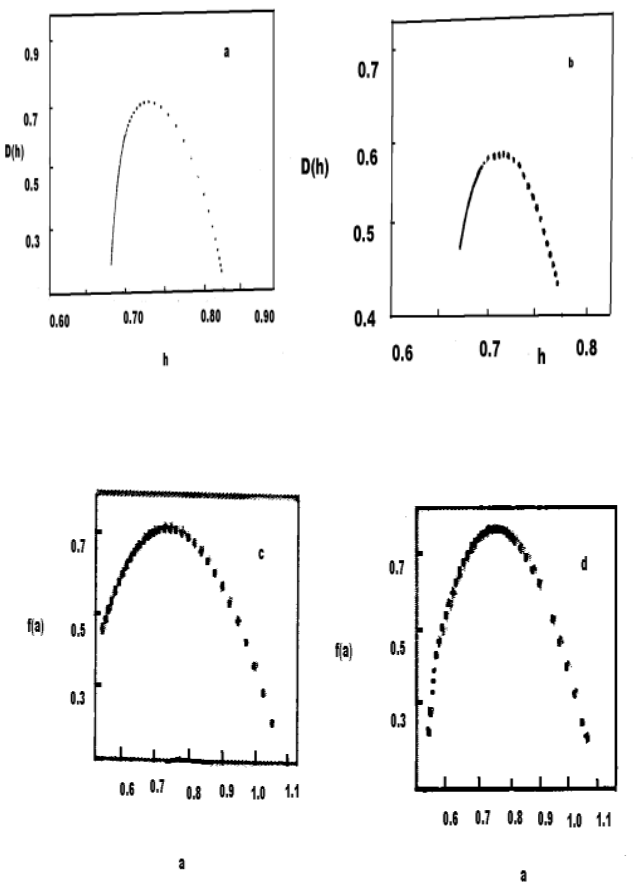

Fig.6, D(h) versus h for for: (a) Solar PV, (b) Wind

Turbine (c) Battery and (d) Inverter.

To investigate the underlying evidence for multi-scaling more carefully, it is necessary to present the statistical findings. for both solar (SPV) and wind turbine(WT) outputs. The support dimensions $\mathrm{D}_{0}=\mathrm{D}_{\max }=\tau(0)$ are $0.65 \& 0.52$ for Solar \& WT respectively, implying capacities of the support measure fractional (i.e., chaotic). The numerical values of the holder exponent for the dimension supports, $\propto\left(D_{\max }\right)$, are 0.7 for SPV and 0.69 for WT (corresponding to $\left.\mathrm{D}(\mathrm{h})_{\max }\right)$, implying the fact that the events with $\propto=\propto,\left(\mathrm{D}_{\max }\right)$ are the most frequent ones. A Hurst exponent of 0.7 and $0 . .69$ describes a very persistent time series and if the distribution is homogenous, there is a unique $\mathrm{h}($ or $\propto)$, but if it is not,, there are several exponents $\mathrm{h}($ or $\propto)$, with the most frequent $\mathrm{h}$ will characterize the series that will play as Hurst exponent.Moreover, $\mathrm{h}^{\mathrm{t}}=\left(\mathrm{h}_{\min }+\mathrm{h}_{\max }\right) / 2$ is almost the same for SPV (0.79) and WT (0.8).This implies that the curves are slightly humped to the left, an effect that is more pronounced for WT than for SPV and a better precision is obtained for the $\mathrm{h}>0$ branch, where the bigger values will prevail (i.e., higher changes 
in demand of electricity, a rare situation). Further, the asymmetrical shape of the spectrum reveals more pronounced in-homogeneities in the events associated with the $\mathrm{q}<\mathrm{h}<0$ branch, associated with smaller values of the power.( Slight change in output more common).This is indicated by $h_{\text {range }}=\left(h_{\max }-h_{\min }\right)$ with the case of larger than the other one. The information dimension, on the other hand for china is $D_{1}=h(q(1))=f(0.7)=0.7$ for which features the scaling behavior of the Information while it is $\mathrm{D}_{1}=\mathrm{h}(\mathrm{q}(1))=\mathrm{h}(0.64)$ for WT.( i.e fractional value for both countries). That is, for both SPV and WT, the available output corresponds to chaotic systems with the problems of forecasting associated with them. The correlation dimension are $\mathrm{D}_{2}=\tau(2)=0.79$ for WT and 0.87 , for SPV, characterizing chaotic attractor, with $\mathrm{D}_{2}>1 / 2$ indicates long range correlations, with the characteristic behavior of adaptivity that plays the role of organizing principle for highly complex, non linear processes that generates fluctuations on a wide range of time scales and, in addition, the lack of characteristic scale helps in preventing excessive mode locking that would restrict the reaction of the event. In short, we can see that, there is longer range correlation for SPV, implying that for the case of an abrupt change of the energy demand, SPV system will have a better answer.

\section{CONCLUSION.}

Kuttumpuzha is one of the most remote villages of Kerala, and is blessed with frequent wind currents making the area suitable for hybrid micro-grid systems in which a major fraction could be met from wind power. For a remote village with low energy demand, a hybrid system can be cost-competitive due to the high costs for a grid extension. Electricity from the grid as such might be cheap, yet the costs for grid extension and the Transmission and Distribution losses connected with it add up to result in a high cost per unit electricity, especially where the grid has to be extended over a considerable distance. Furthermore, the hybrid system has - once it has been implemented - low Operation and Maintenance costs. For these reasons, a stand-alone/macro-grid hybrid system can be the most financially attractive and reliable solution in these cases. In this study, a resource assessment and demand calculation have been carried out and the COE per unit has been ascertained for different systems and configurations.. We found that both RE sources, SPV and WT behave, like most ones in nature, as long term memory phenomena. in both cases the fractal dimension obtained correspond to chaotic processes. Moreover, the correlation dimension gives us an account of long range correlation, with the SPV long range correlated than WT. This lays that SPV power system is better suited to satisfy oscillations in the demand in spite of changes, of $h$ ranges within $0.5<\mathrm{h}<1$, the greater value for WT indicates that the energy output series varies in wider ranges, promising solution to energy expansion in the country along with other alternative resources. Another finding of this study is that a combination of different RETs is better suited for the off-grid electrification of remote villages than the use of one single RES. Further, the differences in the long range correlations, reveals, that power generating system consisting of a combination of $1 \mathrm{KW}$ photo voltaic Array coupled with $1 \mathrm{KW}$ converter along with grid connection is cheap and is better suited to satisfy correlations in the demand.

\section{REFERENCES}

[1] Francois lescaurous 'Dynamics of sectoral energy demand and aggregate intensity economic studies division, October 2010.

[2] Bureau OF energy Efficiency Book,

[3] U. Frish and G.Parisi, in Turbulence and predictability of geophysical flows and Climate Dynamics, edited by M.Ghil,R.Benzi and G.Parisi.(North Holland, New York,1985).

[4] H.G.E Hentechel and I Proccacia, Physica 8D,1983,435.

[5] T.C.Halsey, M.H. Jensen,L.P Kadanoff, I Proccaccia , Phys. Rev A33,1986,1141.

[6] Mallat.s.; A theory for Multi resolution signal decomposition, The wavelet representation.IEEETRANS ,11(7), 1989,674-693.

[7] Mallat.S. and W.L Hwang, IEEE Inform theory ,38,1992,617. 